\title{
The Design and Evaluation of Mobile HCI in Dietary Intake Estimation
}

\author{
Ying-Chieh Liu ${ }^{1, *}$, Chien-Wei Lee ${ }^{1}$, Chien-Hung Chen ${ }^{2}$, and Zhao-Yang Yang ${ }^{3}$ \\ ${ }^{1}$ Industrial Design Department, Chang Gung University, Tao-Yuan, Taiwan \\ yc130@mail.cgu.edu.tw, s923446@gmail.com \\ ${ }^{2}$ Institute for Information Industry, Taipei, Taiwan \\ marken@iii.org.tw \\ ${ }^{3}$ Industrial Design Department, Tatung University, Taipei, Taiwan \\ cyyanattu.edu.tw
}

\begin{abstract}
This research designs and evaluates app interfaces designed to estimate and record dietary intake. Effect and user's preference are taken for consideration. Three potential interfaces are proposed, i.e., a) interactive photo interface; (b) a sketching interface, and c) a resizable shape interface. Trials with 38 university students indicate that a) and b) both provide some useful features, but there is a preference for the interactive photo interface. Future research will focus on design enhancements to improve user experiences in different target groups.
\end{abstract}

Keywords: Mobile HCI, portion size estimation, dietary intake, subjective evaluation.

\section{Introduction}

An increasing number of mobile apps focus on issues related to health care and public health, but few studies have examined the appropriateness and utility of such apps including from a Human-Computer Interaction (HCI) standpoint (Chesanow \& Fogg, 2013). Dietary management is an essential component of weight management and the prevention of clinical diseases (Kuczmarski et al., 1994; Byrd-Bredbenner et al., 2004). A critical issue in effective dietary management is the process of estimating portion sizes. This process typically entails three individual abilities, namely perception, conceptualization and memory, through which the user relates the size or amount of food, consumed to pre-set portion sizes, develops a mental picture of the amount, and recalls the amount of food consumed. This process in frequently subject to errors (Cypel et al., 1997; Slawson \& Eck, 1997; Hunter et al., 1998; Ervin \& SmiciklasWright, 2001; McGuire et al., 2001) which can negate the impact of dietary control or even jeopardize personal health (Arroyo et al., 2007).

Special purpose apps can be designed to provide users with effective and real-time estimates of portion sizes and amounts of unfinished food. Such features could present a significant improvement over current practices which are time-consuming, 
inconvenient and error-prone. This study investigates the design of such innovative aids with subjective user evaluations. Users were asked to use design alternatives with different user interface concepts aiming to determine interface design factors which are conducive to effective use.

\section{Design}

This research adopts an iterative user-centered design process from Bandura's social cognitive theory (1977) to investigate potential design interfaces in the portion estimation process under specific environmental conditions. The design approach entails proposing, evaluating, and selecting design alternatives so as to eventually obtain an effective and user-friendly design. All designs 1) present a selection of predetermined images of foods and associated portions for user consideration, or 2) use handy comparators so users don't need supplemental pocket guides or memory aids to determine an equivalent serving size. One interface design alternative is proposed from the concept of pre-determined images, namely interactive photo interface. Two design alternatives are proposed for the comparators: 1) a sketching-based interface and 2) a shape-resizing interface. Fig. 1 illustrates the sketching-based interface operation. In Fig. 1(a), the user selects a specific food, followed by a comparator (e.g., credit card) in Fig. 1(b). The user then describes the portion size by shading in the appropriate area by scribbling as shown in Fig. 1(c). The user then completes the activity and submits the data for calculation and storage as shown in Fig. 1(e).

In resizing tasks, users select a given initial shape (e.g., circle, ellipse, square) and then baseline the shape against the comparator based on their own experience. The area of the initial shape is enlarged or reduced by dragging its side. The shape resizing task proceeds as follows: Selecting a food item (see Fig. 2(a) and a comparator (Fig. 2(b). In Fig. 2(c), a predefined shape (in this case, a semi-transparent blue square) is superimposed above the comparator. The user drags the shape to indicate the portion size in relation to the comparator and confirms the completed size (Fig. 2(d)). The difference between these two interfaces is that the sketching interface involves a mental picture based on a scribble, while, the shape-based interface allows the user to resize a pre-defined shape by dragging its boundary to modify its shape and size.

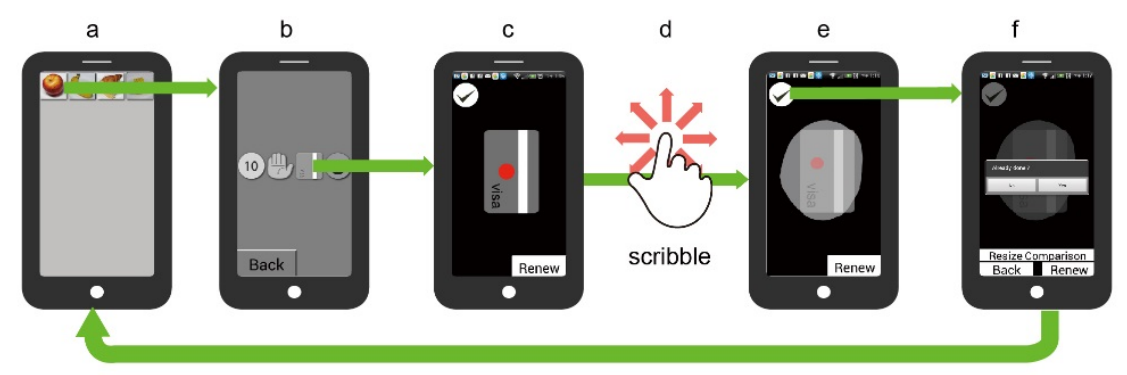

Fig. 1. Sketching interface operation 


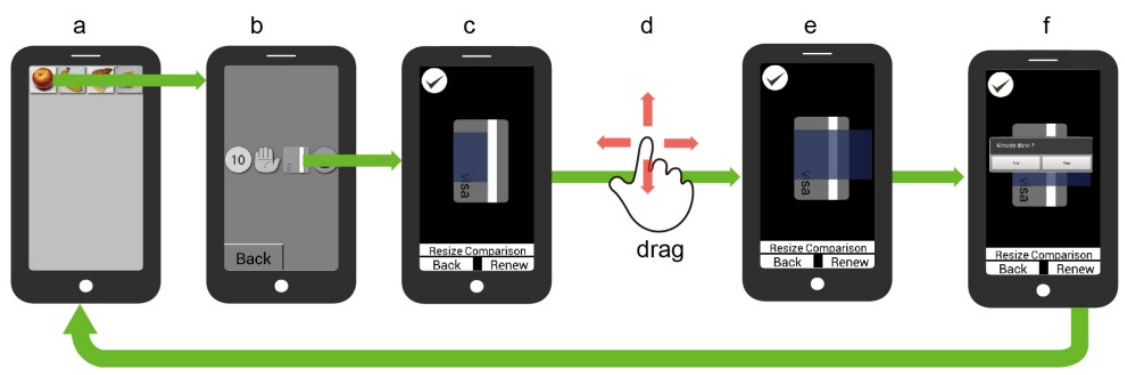

Fig. 2. Shape resizing interface operation

As for the interactive photo, the user selects a picture of the actual food item from a menu (Fig. 3(a)), which is then superimposed on a place setting including a plate, chopsticks and spoon for size reference (Fig. 3(b)). The user then flicks left or right to increase or decrease the size of the food object relative to the size of the place setting objects (Fig. 3(c)).the user first takes a picture of the size of the real food portion next to certain objects. Once the user has found an appropriate size relation, he/she confirms the selection (Fig. 3(d)) and completes the action (Fig. 3(e)).

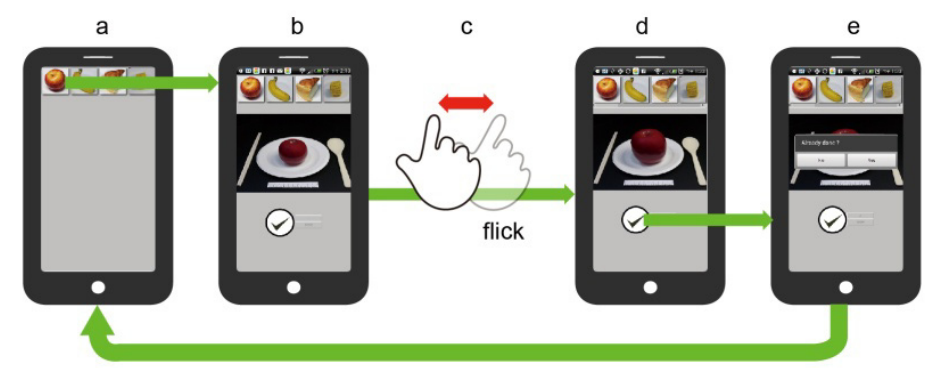

Fig. 3. Interactive photo interface operation

\section{Initial Subjective Evaluation}

An initial subjective evaluation using open-ended questions was conducted to assess interface preference. We recruited 38 students at Chang Gung University in Taiwan, 26 males and 12 females between the ages of 18 and 27 years old. The test group was split into two subgroups to test the different interfaces, with group A (12 females 6 males) testing the interactive photo interface, and group B (14 females, 6 males) testing the sketching interface. Due to time constraints, the resizable interface was not included in this evaluation. A range of 18 food items was selected to ensure consistency and variety (apple, orange, banana, corn, cake, pizza, pork slice, fish, corn flakes, seeds, soya, vegetable, milk, black tea, honey, jam, rice, and tofu). In group A, each subject was asked to visualize a specific food portion using the interactive photo function on a mobile device with a 4.3 inches touch panel. In group B, each subject 
was asked to use the sketching interface. In both groups, all subjects were asked to use their respective interfaces to process all 18 food items a single time. The two groups then switched to the other interface and repeated the evaluation process for five minutes which only allowed subjects to experience the design without repeating the testing of all 18 items. Subjects were then asked to complete a questionnaire with three open-end questions to determine their interface preferences.

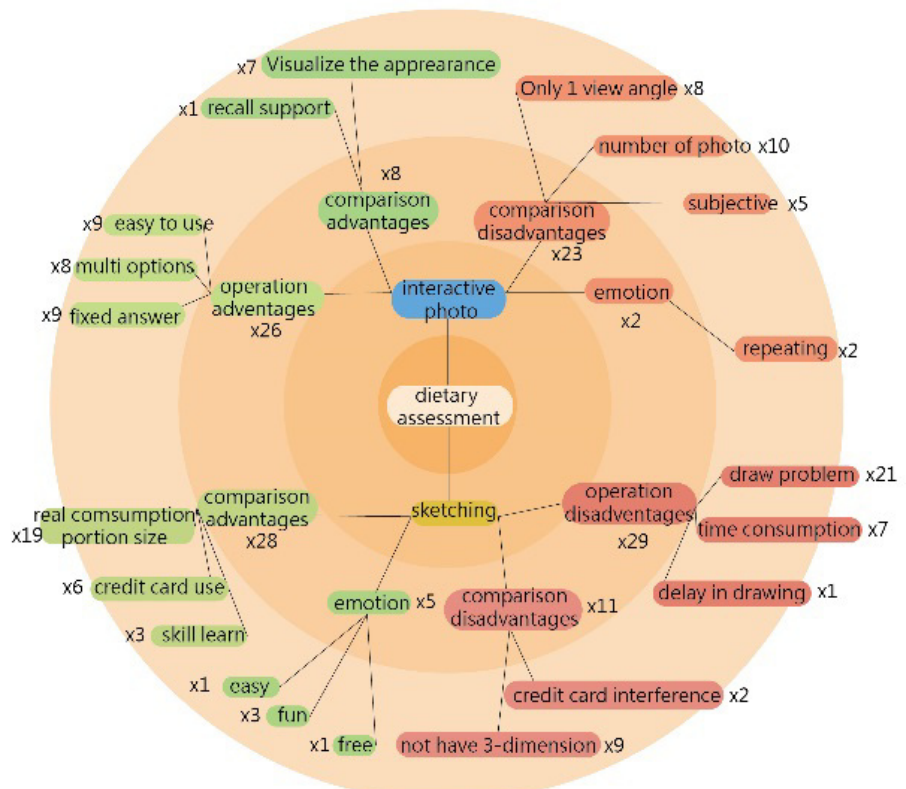

Fig. 4. Thematic network for both interfaces

\section{$4 \quad$ Results}

The thematic network method (Martin \& Hanington, 2013; Lee \& Fielding, 1996) was used to summarize subject response to both interfaces (Fig. 4). Responses dealt with two specific tasks: conducting food portion comparisons and operating the interface. Advantages cited for the interactive photo included ease of use ( 9 respondents), a predefined selection of images to represent specific portion sizes (9), and fixed answers ( 9 subjects). Subjects also indicated concerns regarding the limited selection of food images (10 subjects) and that food images were only portrayed from a single angle (8). As for the sketching interface, most subjects liked that the interface allowed them to draw shapes and sizes that could accurately reflect the food portions (19 respondents) using a handy comparator (6). However, subjects experienced difficulties drawing specific sizes and shapes (21 respondents) and felt the process was time consuming (7). Also, a cost-effective means must be found to expand the variety of food images used in the interactive photo interface. For the sketching interface, operations 
need to be streamlined to reduce the time and effort needed to draw a specific shape. Further testing is also required to objectively evaluate estimation errors and operating time requirements.

\section{References}

1. Arroyo, M., De la Pera, C.M., Ansotegui, L., Rocandio, A.M.: A short training program improves the accuracy of portion-size estimates in future dieti-tians. Arch. Latinoam. Nutr. 57(2), 163-167 (2007)

2. Byrd-Bredbenner, C., Schwartz, J.: The effect of practical portion size measurement aids on the accuracy of portion size estimates made by young adults. Journal of Human Nutrition \& Dietetics 17(4), 351-357 (2004)

3. Chesanow, N., Fogg, B.: Can Health Apps Help Patients Change Their Behavior? (June 2013), http: / /www. medscape.com/viewarticle/806734 (retrieved)

4. Cypel, J., Guenther, P., Petot, G.: Validity of portion- size measurement aids: A review. J. Am. Diet. Assoc. 97, 289-292 (1997)

5. Ervin, R., Smiciklas-Wright, H.: Accuracy in estimating and recalling portion sizes of foods among elderly adults. Nutr. Res., 703-713 (2001)

6. Hunter, D., Sampson, L., Stampfer, M., Colditz, G., Rosner, B., Willett, W.: Variability in portion sizes of commonly consumed foods among a population of women in the United States. Am. J. Epidemiology 127, 1240-1249 (1998)

7. Kuczmarski, M.F., Moshfegh, A., Briefel, R.: Update on nutrition monitoring activities in the United States. Journal of the American Dietetic Association 94(7), 753-760 (1994)

8. Lee, R.M., Fielding, N.: Qualitative Data Analysis: Representations of a Technology: A Comment on Coffey, Holbrook and Atkinson'. Sociological Research Online 1(4), 55 (1996)

9. Martin, B., Hanington, B.: Universal Methods of Design, 1st edn. Rockport, United States of America (2012)

10. McGuire, B., Chambers IV, E., Godwin, S., Brenner, S.: Size categories most effective for estimating portion sizes of muffins. J. Am. Diet. Assoc. 101, 472-474 (2001)

11. Slawson, D.L., Eck, L.H.: Intense practice enhances accuracy of portion size estimation of amorphous foods. J. Am. Diet. Assoc. 97, 295-297 (1997) 